\title{
On accurate simulations of LTE femtocells using an open source simulator
}

\author{
Francesco Capozzi ${ }^{\text {* }}$, Giuseppe Piro ${ }^{2}$, Luigi A Grieco², Gennaro Boggia ${ }^{2}$ and Pietro Camarda ${ }^{2}$
}

\begin{abstract}
Long-term evolution (LTE) femtocells represent a very promising answer to the ever growing bandwidth demand of mobile applications. They can be easily deployed without requiring a centralized planning, to provide high data rate connectivity with a limited coverage. In this way, the overall capacity of the cellular network can be greatly improved. At the same time, the uncoordinated setup of femtocells poses new issues that require a deep and thorough analysis before spreading this technology worldwide. Unfortunately, to the best of our knowledge, no accurate simulation tools are freely available for enabling this kind of investigation. Thus, we present in this study a simulation tool for LTE femtocells, implemented as a module of the emerging open source LTE-sim framework. It encompasses heterogeneous scenarios with both macro and femtocells, spectrum allocation techniques, user mobility, femtocell access policies, and several other features related to this promising technology. After reviewing the status of the art on LTE femtocells, we detail the description of the module that we propose with a major emphasis on the newly devised loss models for indoor scenarios, the new network topology objects, and the most significant enhancements to the simulator protocol stack. Furthermore, to provide a clear understanding of the practical utility of this new simulator, we investigate two indoor and urban scenarios. A scalability test is also presented to demonstrate the efficiency of the proposed tool in terms of processing requirements. All presented results suggest that this new module can be very interesting for the research community, due to its great flexibility and limited computational cost.
\end{abstract}

Keywords: Femtocells, 3GPP, LTE, E-UTRA, Network simulator, Performance evaluation

\section{Introduction}

The capillary diffusion of smartphones and tablets and the integration of connectivity capabilities in common life objects (such as TVs, domestic appliances, and vehicles) are generating a very fast growth of bandwidth demand for mobile applications [1]. Despite emerging broadband technologies (e.g., WiMAX [2] and LTE [3]) are able to enhance the performance of currently used $3 \mathrm{G}$ systems, they might not be able to sustain the expected raise of the traffic volume.

As a consequence, notable improvements in both capacity and coverage of mobile communication systems are required, but they cannot be fulfilled by only enhancing the (PHY) layer and/or increasing the available spectrum. From one side, in fact, latest innovative PHY techniques work very close to the Shannon limit; in this manner,

*Correspondence: francesco.capozzi@itia.cnr.it

1 ITIA-CNR, v. P Lembo, 38F-70124, Bari, Italy

Full list of author information is available at the end of the article they ensure the maximum achievable physical rate. On the other side, the amount of spectrum expected to be licensed during next years is less than what requested by mobile operators [4]. According to this vision, the deployment of small cells within the typical structure of cellular networks, represents the smartest solution for boosting services in these systems [5].

Following this direction, the 3GPP has introduced, within the long-term evolution-advanced (LTE-A) specifications, the possibility to deploy a heterogeneous network (HetNet) composed by macro and small-range (i.e., micro, pico, and femto) cells [6]. Micro and pico cells could be exploited for enhancing coverage and capacity in some regions inside the macrocell. Whereas, femtocells have been devised for offering broadband services in indoor (i.e., home and offices) and outdoor scenarios with a very limited geographical coverage. Among this kind of cells, the role of femtocells become fundamental, because it is

\section{Springer}

(C) 2012 Capozzi et al. : licensee Springer. This is an Open Access article distributed under the terms of the Creative Commons Attribution License (http://creativecommons.org/licenses/by/2.0), which permits unrestricted use, distribution, and reproduction in any medium, provided the original work is properly cited. 
expected that in upcoming years the most part of voice calls and data sessions will take place in home or business environments [7].

A femtocell can be easily set up without any centralized coordination, but simply enabling a low-power and small-range radio base station, which is referred to as home evolved NodeB (HeNB). Such a device has plugand-play capabilities, is connected to the core network through a DSL line, and operates in the spectrum licensed for cellular systems [8]. The uncoordinated nature of femtocell deployment poses novel and interesting challenges on radio resource management (RRM). In fact, classical approaches already adopted in 3G systems to face frequency planning, interference coordination, radio resource scheduling, and access policies could be not useful anymore in HetNet scenarios.

Starting from this premise, it is evident that the development of novel network architectures and the optimization of next generation cellular systems based on femtocells are topics worth of investigation for both industry and academic communities.

In this perspective, the availability of accurate simulation frameworks appears of fundamental importance. Several open-source and commercial tools are today available for simulating some parts of the LTE system [9-14], but none of them provides media access control (MAC) and PHY models for femtocell architectures, handover strategies properly conceived for HetNet, and specific propagation loss models for indoor environments. A system level simulator for LTE femtocells has been proposed in [15]. However, its code is not yet available and, for this reason, it cannot adequately serve the research community. At the present, to the best of our knowledge, the only valuable tool is the one developed for the network simulator 3 (NS-3) within the LENA project [16]. Unfortunately, despite it provides standard compliant definition of the LTE network architecture as well as several propagation loss models for indoor scenarios, at the current status it cannot be sufficient in helping researchers during the study of femtocell related issues. In fact, it does not implement any handover strategies for HetNet, it does not allow the application of different access policies to the HeNB, it offers only a weak support for the quality of service (QoS) management [17] (e.g., only simple scheduling strategies, such as Maximum Throughput and Proportional Fair, have been developed at the MAC layer). In addition, the setup of a realistic simulation scenario is difficult to accomplish, especially for a non-expert NS-3 user.

To bridge this gap, the present study proposes a module that extends the functions of the emerging open source LTE-sim framework [18], thus allowing also the simulation of scenarios with LTE femtocells ${ }^{\mathrm{a}}$. It provides a wide range of features and high scalability. It encompasses scenarios with both macro and femto cells, in multi-cell and multi-user environments, and implements a complete LTE protocol stack along with advanced RRM techniques, such as frequency reuse schemes, packet scheduling, and QoS management. User mobility is also considered and several configurations of mobility patterns and handover mechanisms are considered.

We believe that the developed tool represents a good and useful research tool for tackling several open issues such as: (i) the deployment of heterogeneous LTE networks composed by macro and femto cells; (ii) the design of sophisticated techniques for both radio resource and interference management; (iii) the implementation of novel cognitive-based and/or self-organized algorithms, protocols, and procedures for the setup of next generation broadband networks, and so on.

In order to shed some light on the practical utility of the proposed tool, in this article we report some examples about its application. In particular, some reference indoor and urban scenarios are evaluated. It is worth to note that such an analysis is only intended to demonstrate the general agreement between what is expected from a theoretical point of view and the outcomes of the simulation module we propose.

Furthermore, a scalability analysis to measure the computational requirements of the developed tool (i.e., simulation time and memory usage) has been also provided.

The rest of the article is organized as follows: Section 2 describes LTE femtocells, highlighting pros and cons related to the development of this new technology and the most important open issues that justify the need of a simulation framework. In Section 3, the developed module is described, with particular emphasis on the newly implemented propagation loss models for indoor scenarios, the introduction of new network topology objects, and the enhancements of some modules of the LTE-sim framework. Section 4 provides some reference results on significant scenarios in order to provide a clear assessment of the practical utility of the simulator and to demonstrate its scalability. Finally, Section 5 draws conclusions and forecasts future works.

\section{LTE femtocells}

The LTE system is mainly composed by two parts: the air interface, i.e., the evolved-universal terrestrial radio access network (E-UTRAN), and the packet switched core network, known as Evolved Packet Core. From the network side, the evolved NodeB (eNB) is the only node of the E-UTRAN and it is in charge of providing network connectivity through the air interface to all user equipments (UEs) in the cell, according to the classic cellular network paradigm.

At the physical layer, the radio interface supports both frequency and time divisions duplexing. Channel access, 
instead, is based on orthogonal frequency division multiple access (OFDMA), which provides high flexibility in terms of scheduling and interference management [3].

According to [19], radio resources are allocated in a time/frequency domain. In the time domain, they are distributed every transmission time interval (TTI), each one lasting $1 \mathrm{~ms}$. Furthermore, each TTI is composed by two time slots of $0.5 \mathrm{~ms}$, corresponding to seven OFDM symbols in the default configuration with short cyclic prefix; 10 consecutive TTIs form the LTE Frame lasting $10 \mathrm{~ms}$. In the frequency domain, instead, the whole bandwidth is divided into $180 \mathrm{kHz}$ sub-channels, corresponding to 12 consecutive and equally spaced sub-carriers. A time/frequency radio resource, spanning over one time slot lasting $0.5 \mathrm{~ms}$ in the time domain and over one subchannel in the frequency domain, is called resource block (RB) and corresponds to the smallest radio resource that can be assigned to an UE for data transmission. Note that, due to the fixed sub-channel size, the number of subchannels varies accordingly to different system bandwidth configurations (e.g., 25 and 50 RBs for system bandwidths of 5 and $10 \mathrm{MHz}$, respectively).

In [20], the 3GPP introduced a new low-power and small-range radio based station, i.e., the HeNB, for providing broadband services in indoor and outdoor environments. Such a device is connected to the operator network through a DSL line available at consumers' houses or offices, like a common Wi-Fi access point. The very limited geographical area it covers is called femtocell (Figure 1).

Several benefits are expected from femtocell deployment. First of all, network capacity can be increased. According to [5], OFDMA-based technologies, such as LTE, work very close to the Shannon Limit, and the only way to surely increase the capacity of a single wireless link is to put the transmitter and the receiver closer to each other, as in the rationale of LTE femtocells. In this

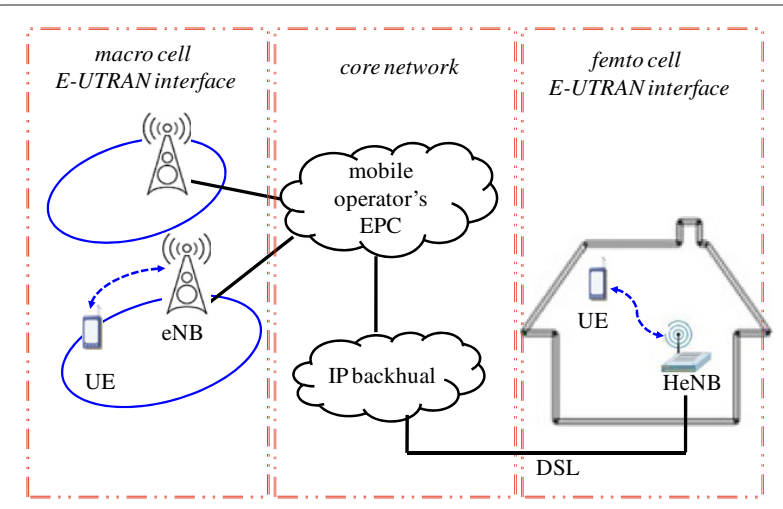

Figure 1 Network architecture macro and femto cells. way, it is also possible to significantly offload the macrocell due to the relevant quota of traffic that femtocells are expected to serve. In addition, from an economical point of view, since an operator can accurately identify the traffic generated/received in each femtocell, it can offer personalized fees and discounts that can be very attractive for consumers.

\subsection{Open issues}

Aforementioned benefits come at the cost of increased complexity in spectrum management. Being unfeasible a centralized frequency planning, in fact, the interference management becomes one of the main issues and new solutions must be found to this regard.

There are several interesting problems that could arise with a wide deployment of LTE femtocells, which have to be carefully accounted for in order to exploit all the potential of this new promising technology.

\subsubsection{How to cope with uncoordinated femtocell deployment?}

An uncoordinated setup of HeNBs, carried out without taking into account the location of macrocells and the bandwidth allocation plan, brings to an increment of the interference level. In these conditions, a mobile operator could not be able to easily apply optimized RRM procedures. We can identify two kinds of interferences: co-layer and cross-layer [21]. The former is produced by a HeNB on users served by other femtocells. The latter, instead, defines the interference between femtocells and macrocells that share the same portion of the spectrum. In this context, the research community is deeply investigating innovative solutions for mitigating the inter-cell interference, furnishing such cells with self-organization and cognitive capabilities [22].

The basic idea is that spectrum assignment should be performed on a distributed basis, by allowing each HeNB to measure the radio environment and to autonomously identify the best frequency bands that minimize the impact of the inter-cell interference.

\subsubsection{Femtocells access policies: open or/and restricted access mode?}

Since femtocells are expected to be deployed in several environments (e.g., home, business, and outdoor scenarios) by both mobile operators and consumers, new issues regarding the network access policy have to be considered.

A typical HeNB deployed by a mobile operator can be setup for working in open access mode (i.e., any connection request can be accepted). In this way, any user, located in its coverage area, can take advantage from the higher capacity offered by the femtocell.

From another side, a femtocell, installed by a consumer for domestic uses, has to operate in restricted access 
mode: the access is allowed only to a limited number of devices. In this case, unauthorized users could suffer the impact of the interference generated by this "private" HeNB [23].

A good tradeoff between the aforementioned approaches is represented by the "hybrid" access method: the $\mathrm{HeNB}$ is in charge of guaranteeing a high performance connection only to a limited number of registered users. At the same time, it could assign a certain amount of radio resources to other users, offering them a minimum service level [24]. A practical example of this policy is the world wide spread FON model, which has been firstly conceived for the WiFi technology [25].

\subsubsection{Radio resource management}

Differently from 3G networks, LTE systems are based on a flattened architecture, with the main consequence that all the RRM procedures have to be handled by the eNB. This aspect becomes very critical for femtocells due to the limited computational capabilities of HeNBs: for instance, there are no guarantees that sophisticated scheduling strategies, such as those proposed in [26-28], could be straightly adopted in LTE femtocells. As a consequence, the research of lightweight RRM techniques remains fully open to novel approaches and methodologies.

\subsubsection{How to handle user mobility?}

Another typical problem of femtocell deployment is strictly connected to user mobility: increasing the number of cells within the same area leads to more and more handover operations. This aspect can become very critical because, despite base stations are connected among them using a dedicated protocol suite (i.e., through the X2 interface), LTE always requires hard handover, which is, in general, a resource-demanding procedure. Also in this case, the research of novel procedures to counteract the impact of handovers would be highly beneficial for the deployment of LTE femtocells [29]. Within this context, some study has been carried out (see, for instance, [30]), but without any explicit reference to femtocells.

\subsubsection{Lacking of a modeling framework}

All the aforementioned open issues are connected to the lack of an unified modeling approach to LTE femtocells. Mainly due to the absence of final specification documents from 3GPP, this means that a common procedure and consequently a common modeling framework to let the researchers properly compare their results are missing.

Therefore, we claim that a flexible simulation platform will support well all the activities of researchers, industries, and standardization bodies that intend to design new network architecture, protocols, and algorithms for next generation broadband systems.

\section{The proposed module}

The module we propose has been implemented within the emerging open source framework LTE-sim[18]. In what follows, we will briefly summarize LTE-sim key aspects and we will describe the details of the newly developed features.

\subsection{Basic background and required upgrades on LTE-sim} LTE-sim is an event-driven simulator written in $\mathrm{C}++$ using the well-known object-oriented paradigm. It encompasses several aspects of LTE networks, including the models of both the E-UTRAN and the evolved packet system, downlink and uplink transmissions, single and multi-cell environments, QoS management, multiusers environment, user mobility, handover procedures, and frequency reuse techniques. Furthermore, the entire LTE protocol stack is framed from the application to the PHY layer, including radio resource control (RRC), radio link control, and MAC entities. This is done for the three kinds of network nodes, that is, UE, eNB, and Mobility Management Entity/Gateway. In addition, the software supports also well-known scheduling strategies (such as Proportional Fair, Modified Largest Weighted Delay First, and Exponential Proportional Fair, Log and Exp rules), Adaptive modulation and coding scheme scheme, channel quality indicator (CQI) feedback, and several other aspects related to the LTE technology.

The high flexibility and modularity of LTE-sim allowed us to devise a complete system for simulating LTE femtocells, built on top of existing features such as application objects, tracing, interaction among macrocells, interference computation, mobility, handover procedures, and so on.

The new module required the upgrade and the addition of classes and functions such as: (i) the HeNB network device; (ii) novel RRC and MAC entities; (iii) dedicated network topology elements (e.g., femtocell, buildings, and streets); and (iv) new channel models.

In line with LTE-sim design criteria, the proposed extension has been freely released under the GPLv3 license [31]. In fact, we believe that the open nature of this software would facilitate the cross-validation and the integration of different approaches proposed by researchers and practitioners working in this field, thus accelerating the progress of the knowledge in this scientific domain.

\subsection{Network devices}

The HeNB device has been created for modeling the base station of a femtocell. Similarly to the eNB, it is identified by an unique ID and its position is defined into a Cartesian system. It keeps track of information related to all registered UEs, like the UE identifier, CQI feedbacks, uplink channel quality, and uplink scheduling request 
(i.e., the amount of data that a user needs to transmit, reported to the target base station through an uplink control message).

A HeNB contains all the entities of the E-UTRAN protocol stack (i.e., RRC, MAC, and PHY) which implementations differ with respect to the eNB. In particular, at RRC and MAC layers, enhanced features have been added, e.g., a new handover procedure more suitable for heterogeneous environments and the possibility to configure the HeNB in both closed and restricted access modes (see Section 3.3 for details).

3GPP specifications do not explicitly define the physical power transmission of a HeNB. However, we set the default value of the maximum transmission power equal to $20 \mathrm{dBm}$, according to suggestions reported in [32]. Obviously, this is not a standardized configuration: the power transmission of the base station could be set taking into account conditions of both surrounding environment and network. To cope with this requirement, LTE-sim allows the user to freely change in a static or dynamical fashion the power transmission of the HeNB, according to its needs.

A slight enhancement has been applied also to the class modeling the UE. With respect to the old LTE-sim implementation, we introduced a boolean flag, updated every time the user position changes. Such a flag is used for recognizing whether a UE is inside or outside a building at a certain time instant; note that this information is fundamental to catch propagation losses due to walls between the transmitter and the receiver.

\subsection{New handover management}

While a user moves along a certain path, the handover manager has the duty of performing cell re-selection and hard handover procedures. It is implemented into the RRC entity as proposed in [33].

In its preliminarily version, LTE-sim supported only a position-based handover procedure, which was used to pick as serving node the closest eNB. In homogeneous scenarios, where all the eNBs transmit at the same power and a space free path loss model can be considered, this kind of technique well approximates a generic policy based on the received power. Obviously, this becomes unfeasible in heterogeneous scenarios like the ones including buildings and femtocells.

For this reason, we conceived a new strategy that for each node selects, as target base station, the one providing the strongest received signal.

Furthermore, in order to cope with several access policies, a special structure is created for the MAC layer of the HeNB. It contains the identifiers of the authorized users (i.e., the so called subscriber group [20]). Accordingly, the handover algorithm allows a given user to join only those cells that either work in open mode or, being in restricted mode, have the considered user in the subscriber group list.

\subsection{New topology objects}

Three new network topology objects have been introduced:

- Femtocell: it has only geometric properties and it is used for detailing the position and the ID of a femtocell.

- Building: it is composed by a number of apartments, each one delimiting the area of a given femtocell. As defined in [34], two different types of building have been developed: (a) Dual Stripe blocks and (b) $5 \times 5$ apartment grid (see Figure 2). The former consists in two buildings composed of two rows of 10 apartments each. The latter, instead, is a building composed of 25 apartments located over a $5 \times 5$ grid. Each building is identified by an unique ID and its position is defined into a Cartesian system. For both

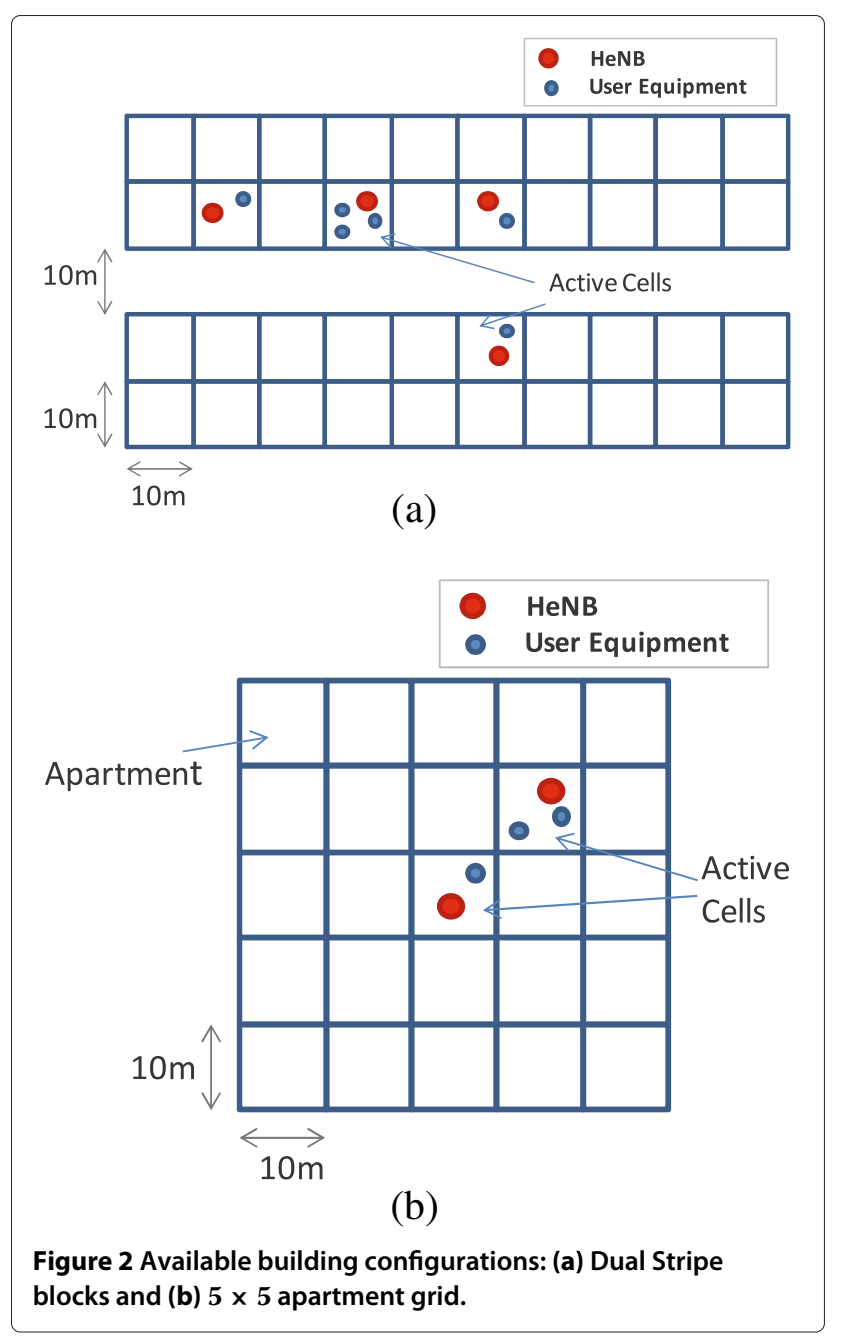


the aforementioned building types, it is possible to define the number of floors. Each apartment has a squared form and an area of $100 \mathrm{~m}^{2}$.

In general, each apartment contains up to one active femtocell, i.e., an active HeNB is working in the femtocell. This means that, for instance, a $5 \times 5$ grid building can contain up to 25 femtocells. The presence of an active femtocell in a single apartment can be randomly decided through the definition of an activity ratio [34], that is the probability that an active home base station is present in an apartment.

- Street: it models two rows of buildings located along a wide road. Its geometric parameters (e.g., the street width, the distance between two adjacent buildings, and the number of building along the street) can be easily customized based on the simulation needs.

As an example, Figure 3 shows a typical urban cross composed of four different streets and $5 \times 5$ grid buildings objects.

In addition, we would like to remark that the developed simulator is flexible and, as a consequence, it can be extended for integrating real street maps. This would be very useful for investigating more realistic environments.

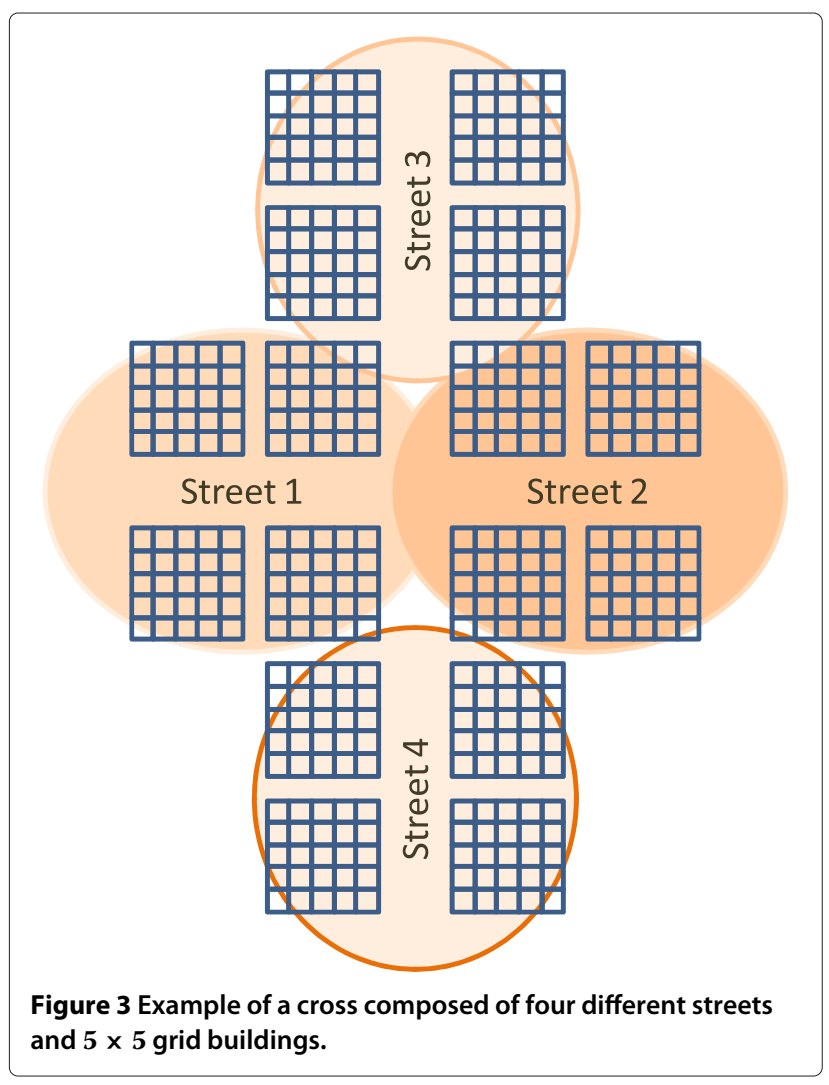

\subsection{Channel models}

The Channel module of the simulator handles packet transmissions and models the propagation loss by means four different phenomena as suggested in [35]: (i) the path loss, (ii) the penetration loss, (iii) the shadowing, and (iv) the fast fading due to the signal multipath.

For a user located in a building and served by the base station of a macrocell, the path loss calculation takes in consideration also an additional attenuation factor due to the presence of an external wall (default value of the external wall attenuation is $20 \mathrm{~dB}$ [34]).

Moreover, in order to cope with the peculiar features of femtocells, two new path loss models have been introduced. The first one is the indoor propagation model defined in [34] for a dense urban deployment of femtocells. It evaluates the path loss, $P_{L}$, considering only the distance, $R$, between the transmitter and the receiver expressed in meters:

$$
P_{L}[\mathrm{~dB}]=127+30 \cdot \log _{10}(R / 1000) .
$$

The second one has been developed within the WinnerII project for indoor residential environments [36]. It offers a high accuracy at the cost of an increased computational complexity:

$$
P_{L}[\mathrm{~dB}]=A \cdot \log _{10}(R)+B+C \cdot \log _{10}\left(\frac{f_{c}}{5}\right)+X
$$

where $R$ is expressed in meters; the central frequency $f_{c}$ is expressed in gigahertz; the values of other parameters $A, B$, and $C$ depend on the number of walls and floors between the transmitter and the receiver.

It is important to remark that the quality of the signal received by users inside a building depends on how the bandwidth is shared among femtocells. To investigate this concept, we compared the distribution of signalto-interference plus noise ratio (SINR) provided by the WinnerII channel model inside a $5 \times 5$ apartment grid, supposing to assign the spectrum by means of the reuse- 1 (i.e., all femtocells share the same bandwidth of $20 \mathrm{MHz}$ ) and the reuse- $1 / 2$ scheme (i.e., two portions of the spectrum, of $10 \mathrm{MHz}$ each, are distributed among HeNBs imposing that two adjacent femtocells cannot work on the same operative bandwidth). From Figure 4, it is evident that the adoption of a frequency reuse scheme improves the channel quality perceived by users. In fact, by distributing the whole spectrum among HeNBs, it is possible to reduce the interference level. However, this advantage could be reached at the cost of the network throughput (e.g., the higher the number of portions of the spectrum considered during the frequency planning strategy, the lower is the bandwidth available for each femtocell). Such aspects will be better discussed in Section 4.1. 


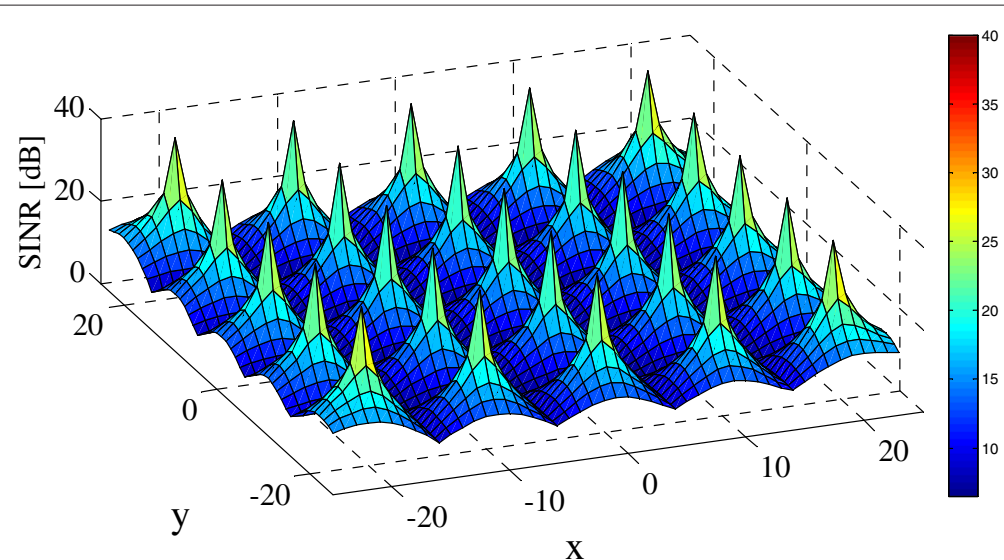

(a)

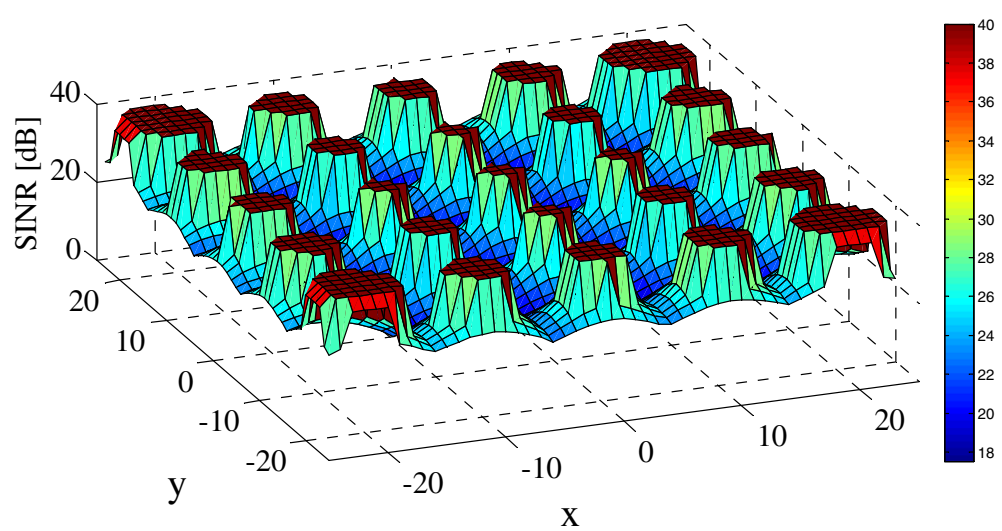

(b)

Figure 4 SINR measured inside a building with: (a) no frequency reuse and (b) reuse-1/2 scheme.

\section{Working with the proposed module}

In this section, we provide an overview about some of the possible studies that can be carried out using our simulation framework. To this end, the impact of co-layer interference in indoor environments generated among HeNB as well as the performance gains coming from femtocell deployment in an urban scenario have been analyzed. At last, a scalability study about computational requirements (i.e., simulation time and memory usage) of the simulator is also provided. Simulation parameters are summarized in Table 1.

\subsection{Studying the impact of co-layer interference in an indoor environment}

The impact that the co-layer interference due to the communication between home base station and user has been evaluated in an indoor scenario composed by a single $5 \times 5$ apartment grid.

The analysis has been carried out by considering three different frequency reuse schemes, e.g., the reuse- 1 , the reuse- $1 / 2$, and the reuse- $1 / 4^{\mathrm{b}}$, and by imposing to have in each apartment 8 UEs receiving at the same time a downlink traffic mix composed by one video flow, one VoIP flow, and one best effort flow.

For the video application, we used a traffic trace obtained from a test sequence (i.e., "foreman.yuv") available at [37]. The original sequence (at 25 frame/s, CIF resolution $352 \times 288$, and YUV format) has been firstly repeated for the whole simulation time. Then, the obtained video has been compressed using H.264 standard compression at the average coding rate of $440 \mathrm{kbps}$. Instead, for G.729 voice flows, we adopted an ON/OFF Markov model, where the ON period is exponentially distributed with mean value $3 \mathrm{~s}$, and the OFF period has a truncated exponential pdf with an upper limit of $6.9 \mathrm{~s}$ and an average value of $3 \mathrm{~s}$ [38]. During the ON period, the source sends 20 bytes long packets every $20 \mathrm{~ms}$ (i.e., the source data rate is $8 \mathrm{kbps}$ ), while during the OFF period the rate is zero because we assume the presence of a voice activity detector. Finally, for the best effort flows we have considered infinite buffer sources.

For all the aforementioned spectrum configurations, we compared the behavior of well-known packet schedulers: the proportional fair (PF) [3], the logarithmic (LOG) rule 
Table 1 Simulation parameters

\begin{tabular}{|c|c|c|c|}
\hline Parameter & Indoor environment & Urban scenario & Scalability test \\
\hline Total bandwidth & \multicolumn{3}{|c|}{$20 \mathrm{MHz}$} \\
\hline eNB power transmission & \multicolumn{3}{|c|}{$43 \mathrm{dBm}$, equally distributed among sub-channels } \\
\hline HeNB power transmission & \multicolumn{3}{|c|}{$20 \mathrm{dBm}$, equally distributed among sub-channels } \\
\hline CQI & \multicolumn{3}{|c|}{ Full bandwidth and periodic reporting scheme. Measured period: 2 ms. } \\
\hline Apartment size & $100 \mathrm{~m}^{2}$ & $100 \mathrm{~m}^{2}$ & $100 \mathrm{~m}^{2}$ \\
\hline \# apartments in a building & 25 & 25 & 25 \\
\hline Radius of the macrocell & N.A. & $500 \mathrm{~m}$ & $500 \mathrm{~m}$ \\
\hline \# buildings & 1 & 56 & from 1 to 6 \\
\hline Frequency Reuse Scheme & reuse- 1 , reuse- $1 / 2$, and reuse- $1 / 4$ & reuse-1 & reuse-1 \\
\hline \# users & 8 per HeNB & from 40 to 120 & from 0 to 300 \\
\hline Scheduler & PF, LOG rule, and FLS & PF & PF \\
\hline Traffic & Video, VoIP, and best-effort & best-effort & best-effort \\
\hline
\end{tabular}

[39], and the frame level scheduler (FLS) $[40,41])$. In particular, network performance has been evaluated with reference to the throughput achieved by best effort flows and the packet loss rate (PLR) of multimedia ones (i.e., video and VoIP).

From Figure 5 it is possible to note that the throughput of best effort flows can be maximized using the reuse$1 / 2$ scheme, which is able to provide the best trade-off between the bandwidth available in each femtocell and the interference level produced by HeNBs inside the building. The adoption of the reuse- $1 / 4$ scheme produces always the worst network performance. In fact, despite enormous benefits that the reuse-1/4 scheme offers in terms of SINR (due to the very limited level of the co-layer interference), it imposes that each HeNB uses a small bandwidth, thus decreasing the overall achievable system throughput.
To gain a further insight, we evaluated the Jain fairness index [42], finding that it is higher than 0.8 in all considered conditions. This means that neither the frequency reuse strategy nor the scheduling algorithm affects the fairness in bandwidth sharing of best effort flows.

Figure 6 reports the PLR of video flows, showing that, the reuse- $1 / 2$ is able to provide the best QoS.

Moreover, in line with results presented in [40], the FLS approach, which has been designed for guaranteeing bounded delays to multimedia flows, provides the lowest PLR. Thus, it ensures the highest video quality to mobile users. This performance gain comes at the expense of performance experienced by best effort flows, which a smaller amount of bandwidth is assigned to when FLS is use (see Figure 5).

With reference to VoIP flows, we observed that they achieved smaller PLR values than the ones related to

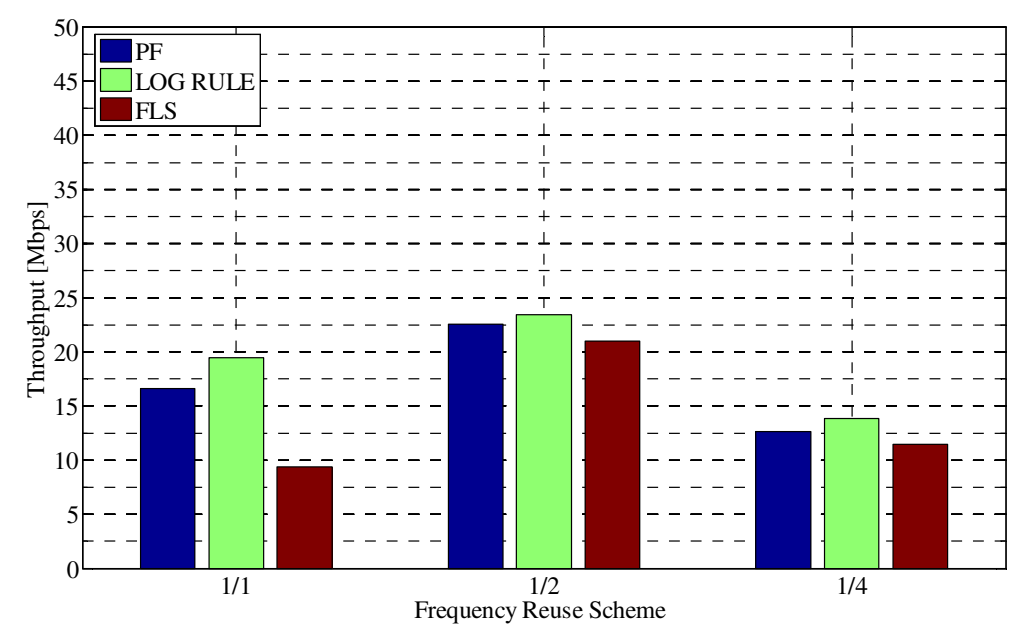

Figure 5 Aggregate cell throughput for best effort flows with different frequency reuse schemes. 


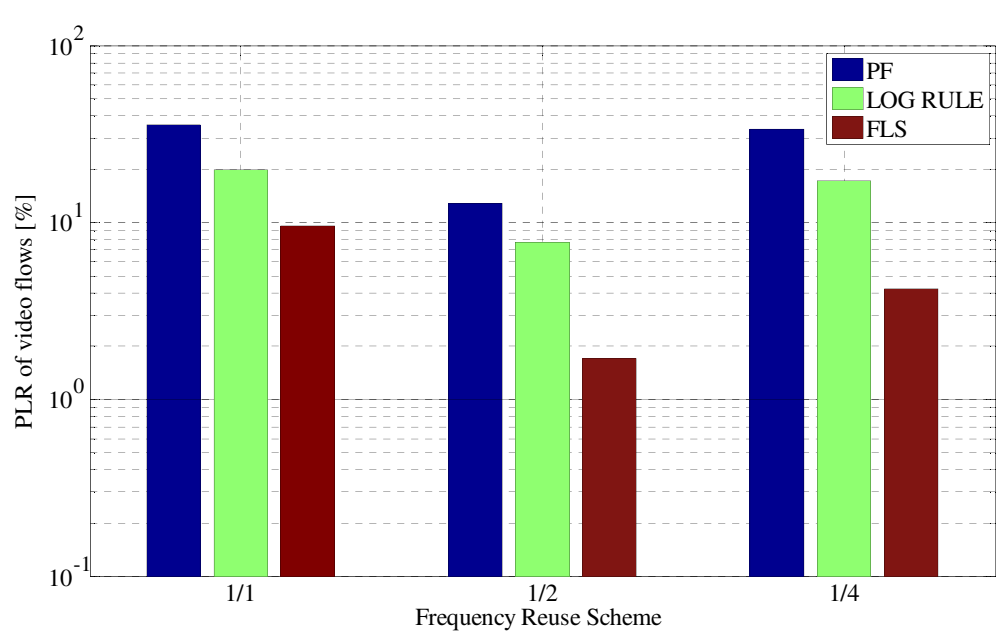

Figure 6 PLR of video flows with different frequency reuse schemes.

videos without any noticeable differences among all considered scheduling approaches (the PLR is less that $5 \%$ when the reuse- 1 scheme is used; $0 \%$ otherwise). The reason is that such applications get a higher priority from the scheduler because they have a lower source bit rate.

We can conclude that the typical problem connected to interference management, as already explained in Section 2, lies in the unpredictability of the deployed scenario. In fact, in a dense urban environment the network operator could not have enough information about the femtocell density and the HeNBs positions in each building. Thus, the possibility to apply any kind of frequency planning strategy vanishes. Therefore, the proposed scenario is usually considered for investigating the impact of different dynamic spectrum allocation policies for interference avoidance in femtocell environments, and the proposed analysis represents a valid starting point for studies in this direction.

\subsection{Capacity enhancement in urban environment}

Now, we investigate the impact of the femtocell deployment in urban environments. To this end, we designed a scenario consisting of one macrocell and 56 buildings located as in a typical urban cross (see Figure 7).

The eNB is located at the center of the macrocell and it transmits using an omni-directional antenna in a $20 \mathrm{MHz}$ bandwidth. Two different cases are object of the study:

- traditional urban environment without femtocells where only one macrocell and buildings are used as reference case;

- urban environment with femtocells where one femtocell per apartment is assumed to be active and working on the same operative bandwidth of the macrocell.
Simulations were carried out varying the number of users, each one receiving a downlink flow modeled with an infinite buffer source.

Figure 8 shows the average user throughput of outdoor and indoor users.

In the typical urban scenario without femtocells, indoor users will be anyway served by the macrocell suffering for low channel quality due to the strong influence of wall attenuation. Introducing femtocells inside the buildings, indoor users will experience significant increase in terms of throughput. Performance of outdoor users, on the other hand, should not take any advantage in terms of experienced SINR. In fact, when home base stations and eNBs share the same bandwidth, outdoor users located nearby the building will sense high level of interference with consequent performance losses. Nevertheless,

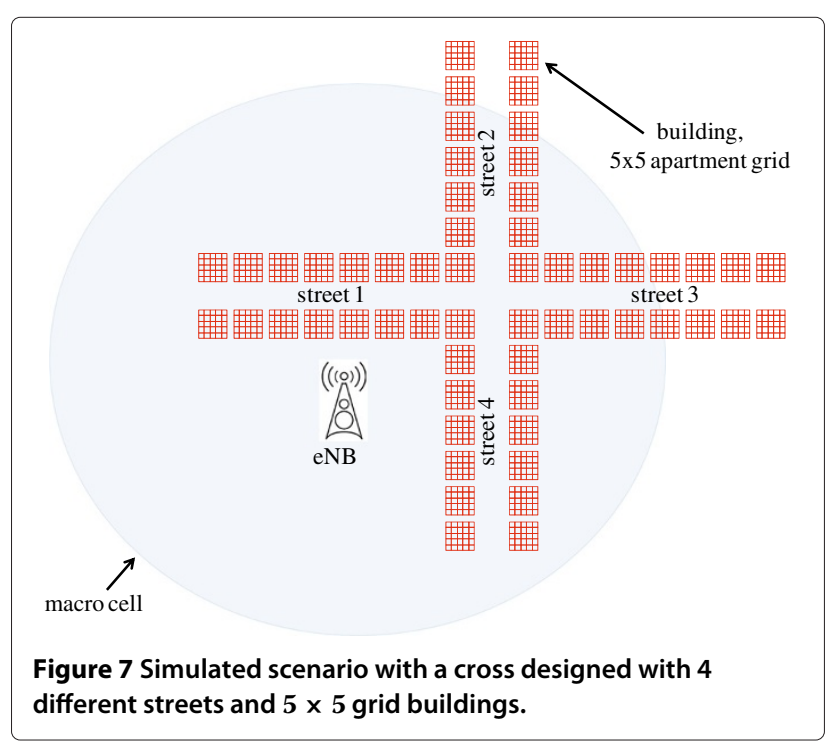




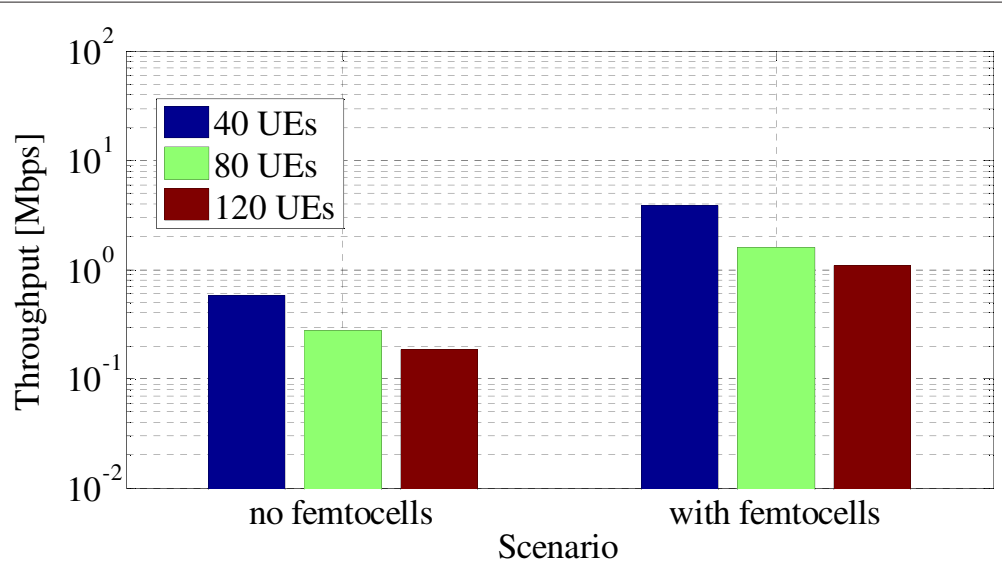

(a)

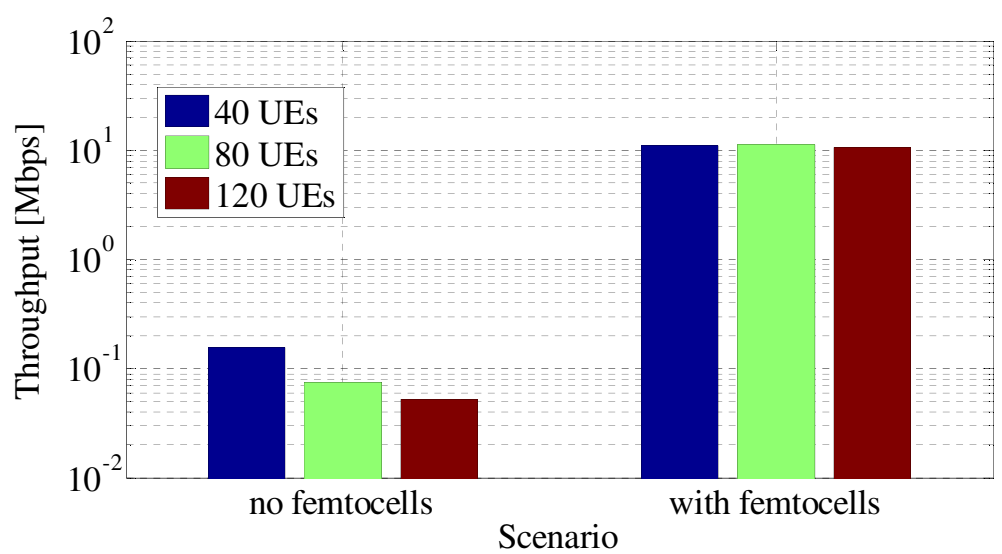

(b)

Figure 8 Average throughput for: (a) outdoor and (b) indoor users.

Figure 8 demonstrates that performance of outdoor users improve as well. This apparently counter intuitive result is mainly due to the macrocell unloading effect contributed by the introduction of femtocells (see Section 2). In other words, a certain number of users is served by HeNBs and the amount of resources available for remaining outdoor users consequently increases.

Finally, Figure 9 shows that the overall system capacity can be greatly improved thanks to the adoption of femtocells.

We highlight, once again, that the proposed studies aim at showing how our module can be easily used to investigate the main issues related to the deployment of femtocells. We think that, starting from this basic scenario, several novel techniques can be tested and compared.

\subsection{Scalability test}

As final aspect, we propose a scalability analysis of the proposed module in terms of both simulation time and memory usage on a Linux machine with a $2.6 \mathrm{GHz} \mathrm{CPU}$ and 4 GBytes of RAM.
The investigated LTE network is composed of one macrocell and a number, $N$, of buildings, with $N$ ranging from 1 to 6 . Buildings are uniformly distributed inside the macrocell and, in each of them, 25 femtocells are enabled. Simulations have been carried out considering a fixed number of UEs equal to 30 that belong to the macrocell and varying the number of UEs in each building in the range [10-50]. One active downlink flow with infinite buffer characteristics is considered per each UE. Moreover, a simulation time of $30 \mathrm{~s}$ is considered. With these conditions, the "lightest" scenario will be composed of 26 cells and 40 users, whereas the "heaviest" one will embrace 151 cells and 330 users.

The choice to use infinite buffer applications (i.e., there is always a packets to transmit) is useful to evaluate the simulator behavior under strenuous conditions.

Figure 10 shows time and memory required in the aforementioned scenarios. Program execution time linearly increases with both the number of buildings and the number of users. Nevertheless, it remains limited also in scenarios with the highest computational load. 


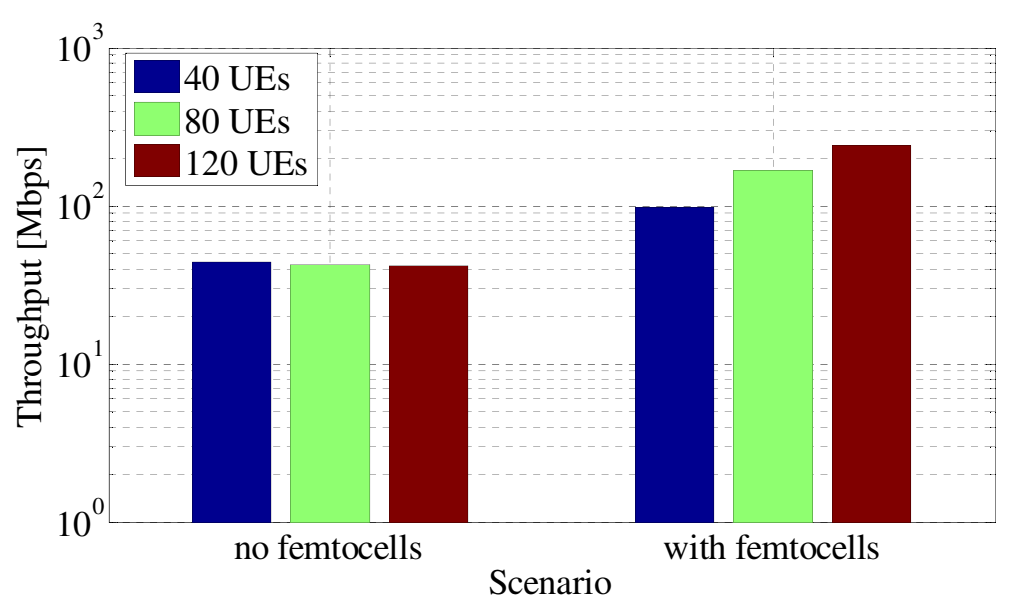

Figure 9 Aggregated throughput.

Different considerations can be made for the memory usage. Despite it increases as the number of buildings increases, it settles as soon as the number of users exceeds a certain value (i.e., about 25 users per building), and this is a nice property for the simulator.

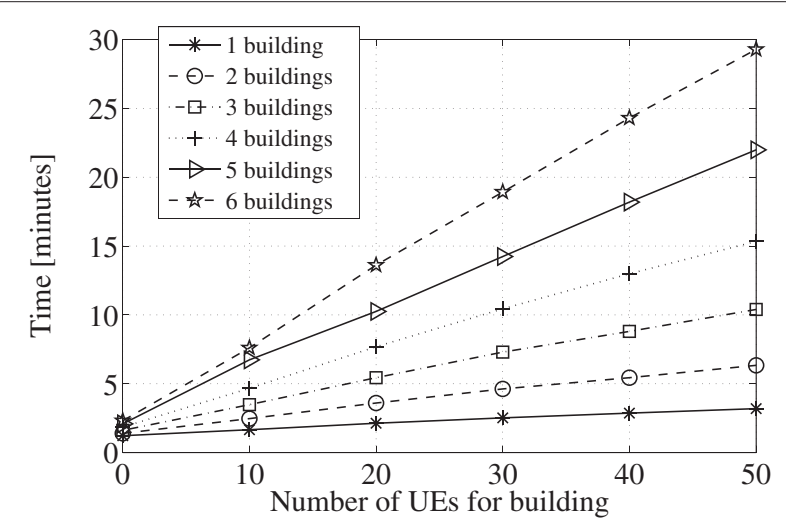

(a)

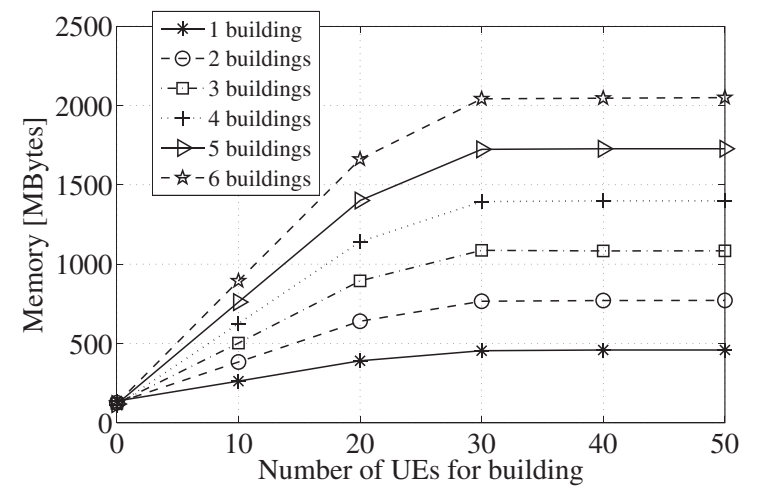

(b)

Figure 10 Simulator performance in terms of (a) simulation time and (b) memory usage.

\section{Conclusions}

In this article, we presented a novel module for simulating LTE femtocells within the LTE-sim open-source framework. We conclude our study by providing a final critical analysis on the presented tool, possibly remarking its pros and cons.

First of all, we have demonstrated that the developed simulator offers the possibility to investigate performances in a wide range of scenarios that can differ in terms of network layouts (i.e., number and position of base stations, building, and streets), system load (i.e., number of users and applications), user mobility, physical settings, handover and access policies strategies, and so on. It is important to note that all implemented features and models have been devised in order to make the simulation framework compliant with 3GPP specifications.

Unfortunately, it presents some lacks. If we focus the attention on available topology entities, it is easy to understand that indoor scenarios different from those described by the 3GPP cannot be directly simulated with the current version of LTE-sim. A user that wants to test more complex urban scenarios is forced to extend the framework by implementing its specific network settings.

Similar considerations can be carried out for all the implemented radio resource management techniques (e.g., scheduling, handover and interference management, cell selection procedures, and so on). Also in this case, we note that the simulator offers only few simple approaches, i.e., those described in this manuscript and in [18], thus leaving the user free to implement more sophisticated solutions. Furthermore, we believe that the extreme modularity of the tool could make the development of complex radio resource allocation schemes (like those discussed in [43]), as well as other algorithms and protocols based on both self-organizing network (SON) and cognitive 
paradigms, not too difficult to accomplish for any LTE practitioner.

We have to remark also that LTE-sim has been originally conceived for simulating only LTE networks. Hence, possible interactions among LTE and other wireless technologies (e.g., WiFi, WiMAX, and other $3 G$ cellular systems) cannot be (for the time being) evaluated with the presented tool. On the other hand, LTE-sim has been designed as a single-threaded software and it is not able to exploit the parallel computational power provided by multi-core architectures. For this reason the evaluation of big cellular configurations would require very high simulation times, thus slowing the research activity in this field.

Anyway, despite these limitations, we claim that our module represents a fundamental tool for researchers interested in developing innovative solutions in the field of radio resource management in next generation cellular networks.

As a future study, we plan to enhance the entire LTE-sim framework with the aim of fixing all aforementioned gaps: not only missing features will be gracefully added (also with the help of research laboratories that are actively supporting LTE-sim upgrades), but also a multi-thread extension and a framework to handle interoperability with wireless technologies other than LTE will be deployed.

\section{Endnotes}

${ }^{\mathrm{a}} \mathrm{A}$ preliminary version of this work has been presented in [44] Additional file 1.

b When the reuse- $1 / 4$ is applied, the entire $20 \mathrm{MHz}$ bandwidth is partitioned in four portions. Then, femtocells are grouped in squared clusters composed by four femtocells and each portion of the spectrum is assigned to only one HeNB belonging to a given cluster.

\section{Additional file}

Additional file 1: A system-level simulation framework for LTE Femtocells.

\section{Competing interests}

The authors declare that they have no competing interests.

\section{Acknowledgements}

This study was partially funded by the Apulia Region Project PS 025 (ICT supporting logistic services: a model of organized market) and the National Operative Program ERMES (Enhance Risk Management through Extended Sensors). This study was done when Francesco Capozzi was at "DEE-Dip. Di Elettrotecnica ed Elettronica", Politecnico di Bari.

\section{Author details}

${ }^{1}$ ITIA-CNR, v. P Lembo, 38F-70124, Bari, Italy. ${ }^{2}$ Dip. di Elettrotecnica ed Elettronica, Politecnico di Bari, v. Orabona, 4-70125, Bari, Italy.

Received: 1 June 2012 Accepted: 26 September 2012

Published: 30 October 2012

\section{References}

1. Cisco, Cisco Visual Networking Index: Global Mobile Data Traffic Forecast Update, 2010-2016. (Cisco White Papers, 2012). [OnLine] Available: [http:// www.cisco.com/en/US/solutions/collateral/ns341/ns525/ns537/ns705/ ns827/white_paper_c11-520862.pdf]

2. L Nuaymi, WiMAX: Technology for Broadband Wireless Access. (Wiley, New York, 2008)

3. E Dahlman, S Parkvall, J Skold, P Beming, 3G Evolution HSPA and LTE for Mobile Broadband. (Academic Press, USA, 2008)

4. Onyeije Consulting LLC, Solving the capacity crunch (2011)

5. V Chandrasekhar, J Andrews, A Gatherer, Femtocell networks: a survey IEEE Commun. Mag. 46(9), 59-67 (2008)

6. A Damnjanovic, J Monotjo, Y Wei, T Ji, T Luo, M Vajapeyam, T Yoo, O Song, D Malladi, A survey on 3GPP heterogeneous networks. IEEE Wirel. Commun. 18(3), 10-21 (2011)

7. The Femto Forum, The Best That LTE Can Be - Why LTE Needs Femtocells, Femto Forum White Paper (2010)

8. P Lin, J Zhang, Y Chen, Q Zhang, Macro-femto heterogeneous network deployment and management: from business models to technical solutions. IEEE Wirel. Commun. 18(3), 64-70 (2011)

9. C Mehlführer, M Wrulich, JC Ikuno, D Bosanska, M Rupp, in Proc. of European Signal Processing Conf., EUSIPCO. Simulating the long term evolution physical layer, (Glasgow, Scotland, 2009), pp. 1472-1478

10. JC Ikuno, M Wrulich, M Rupp, in Proc. of IEEE Veh. Technol. Conf., VTC Spring. System level simulation of LTE networks, (Taipei, Taiwan, 2010), pp. 1-5

11. S Ascent, 3GPP LTE toolbox and blockset. [OnLine] Available: [http:// www.steepestascent.com/content/default.asp?page=s2_10]

12. mimoOn, mi!Mobile. [OnLine] Available: [http://www.mimoon.de/pages/ Products/miMobile/

13. Aricent, LTE layer 1 - LTE baseband/PHY library. [OnLine] Available: [http:// www.aricent.com/Expertise/LTE.aspx]

14. G Piro, N Baldo, M Miozzo, in Proc. of Int. Conf. on Simulation Tools and Techniques, SIMUTools. An LTE module for the ns-3 network simulator, (Barcelona, Spain, 2011), pp. 415-422

15. M Simsek, T Akbudak, B Zhao, A Czylwik, in Proc. of Int. Workshop on Smart Antennas, WSA. An LTE-femtocell dynamic system level simulator, (Duisburg, Germany, 2010), pp. 66-71

16. CTTC and Ubiquisys, LTE-EPC Network Simulator (LENA). [OnLine] Available: [http://iptechwiki.cttc.es/LTE-EPC_Network_Simulator_(LENA)]

17. G Piro, LA Grieco, G Boggia, P Camarda, QoS in wireless LAN: a comparison between feedback-based and earliest due-date approaches. Elsevier Comput. Commun. 35(3), 298-308 (2012)

18. G Piro, L Grieco, G Boggia, F Capozzi, P Camarda, Simulating LTE cellular systems: an open-source framework. IEEE Trans. Veh. Technol. 60(2), 498-513 (2011)

19. 3GPP, Tech. Specif. Group Radio Access Network - Physical Channel and Modulation (Release 8), 3GPP TS 36.211 (2008)

20. 3GPP, Tech. Specif. Group Radio Access Network - Service requirements for Home Node B (HNB) and Home eNode B (HeNB), 3GPP TS 22.220 (2011)

21. The Femto Forum, Interference Managament in OFDMA Femtocells, Femto Forum White Paper (2010)

22. D Lopez-Perez, A Valcarce, G de la Roche, J Zhang, OFDMA Femtocells: A roadmap on interference avoidance. IEEE Commun. Mag. 47(9), 41-48 (2009)

23. H Mahmoud, I Guvenc, in Proc. IEEE Int. Symposium on Personal, Indoor and Mobile Radio Communications, PIMRC. A comparative study of different deployment modes for femtocell networks, (Palo Alto, USA, 2009), pp. 1-5

24. G de la Roche, DD Lopez-Perez, J Zhang, Access control mechanisms for femtocells. IEEE Commun. Mag. 48(1), 33-39 (2010)

25. FON, A word of free wi-fi. [OnLine] Available: [http://www.fon.com/]

26. C Stocchi, N Marchetti, N Prasad, in Proc. of Wireless Communication, Vehicular Technology, Information Theory and Aerospace Electronic Systems Technology Conf., Wireless VITAE. Self-optimized radio resource management techniques for LTE-A local area deployments, (Aalborg, Denmark, 2011), pp. 1-5

27. Y Chen, Z Feng, P Zhang, Y Li, Q Zhang, L Tan, in Proc. of Wireless Commun. and Netw. Conf., WCNC. Complete interference solution with MWSC consideration for OFDMA macro/femtocell hierarchical networks, (Beijing, China, 2011), pp. 2191-2196

28. F Tariq, L Dooley, A Poulton, J Yusheng, in Proc. of Wireless Commun. and Mobile Comput. Conf., IWCMC. Dynamic fractional frequency reuse based 
hybrid resource management for femtocell networks, (Milton Keynes, UK, 2011), pp. 272-277

29. DM Rose, T Jansen, T Kurner, in Proc. of IEEE Veh. Technol. Conf., VTC Spring. Modeling of Femto Cells - Simulation of Interference and Handovers in LTE Networks, (Braunschweig, Germany, 2011), pp. 1-5

30. K Dimou, M Wang, Y Yang, M Kazmi, A Larmo, J Pettersson, W Muller, Y Timner, in Proc. of IEEE Veh. Technol. Conf., VTC Fall. Handover within 3GPP LTE: Design Principles and Performance, (Stockholm, Sweden, 2009), pp. 1-5

31. G Piro, LTE-Sim - the LTE simulator. [OnLine] Available: [http://telematics, poliba.it/LTE-Sim]

32. 3GPP, Tech. Specif. Group Radio Access Network - LTE Home Node B downlink simulation results with flexible Home Node B power 3GPP TSG-RAN WG4 R4-071540 (2007)

33. 3GPP, Tech. Specif. Group Radio Access Network - Evolved Universal Terrestrial Radio Access (E-UTRA) and Evolved Universal Terrestrial Radio Access Network (E-UTRAN); Radio Resource Control (RRC); Protocol specification (Release 9), 3GPP TS 36.331 (2009)

34. 3GPP, R4-092042, Simulation assumptions and parameters for FDD HeNB RF requirements, 3GPP TSG RAN WG4 Meeting 51 (2009)

35. 3GPP, Tech. Specif. Group Radio Access Network; Physical layer aspect for evolved Universal Terrestrial Radio Access (UTRA) (Release 7), 3GPP TR 25.814 (2006)

36. IST-Winner II, WINNER II Channel Models - Deliverable D1.1.2 V1.2 (2007) [OnLine] Available: [http://www.ist-winner.org/WINNER2-Deliverables/ D1.1.2v1.2.pdf]

37. Video test sequence. [OnLine] Available: [http://www.hlevkin.com/]

38. G Boggia, P Camarda, LA Grieco, S Mascolo, Feedback-based Control for Providing Real-time Services with the 802.11e MAC. IEEE/ACM Trans. Netw. 15(2), 323-333 (2007)

39. B Sadiq, R Madan, A Sampath, Downlink scheduling for multiclass traffic in LTE. EURASIP J. Wirel. Commun. Netw. 2009, 1-18 (2009)

40. G Piro, L Grieco, G Boggia, R Fortuna, P Camarda, Two-level downlink scheduling for real-time multimedia services in LTE networks. IEEE Trans. Multimedia. 13(5), 1052-1065 (2011)

41. G Piro, LA Grieco, G Boggia, P Camarda, in Proc. of European Wireless, EW. A Two-level Scheduling Algorithm for QoS Support in the Downlink of LTE Cellular Networks, (Lucca, Italy, 2010), pp. 246-253

42. R Jain, The Art of Computer Systems Performance Analysis. (John Wiley \& Sons, New York, 1991)

43. F Capozzi, G Piro, LA Grieco, G Boggia, P Camarda, Downlink packet scheduling in Ite cellular networks: Key design issues and a survey. IEEE Commun. Surv. Tuts. (Early Access Articles 2012)

44. F Capozzi, G Piro, LA Grieco, G Boggia, P Camarda, in Proc. of International ICST Conference on Simulation Tools and Techniques, SIMUTools. A system-level simulation framework for LTE Femtocell, (Desenzano, Italy, p. 2012

\section{Submit your manuscript to a SpringerOpen ${ }^{\circ}$ journal and benefit from:}

- Convenient online submission

- Rigorous peer review

- Immediate publication on acceptance

- Open access: articles freely available online

- High visibility within the field

- Retaining the copyright to your article

Submit your next manuscript at $\boldsymbol{\nabla}$ springeropen.com 